\title{
Pion Propagation near the QCD Chiral Phase Transition
}

\author{
D. T. Son ${ }^{1,3}$ and M. A. Stephanov ${ }^{2,3}$ \\ ${ }^{1}$ Physics Department, Columbia University, New York, New York 10027 \\ ${ }^{2}$ Department of Physics, University of Illinois, Chicago, Illinois 60607-7059 \\ ${ }^{3}$ RIKEN-BNL Research Center, Brookhaven National Laboratory, Upton, New York 11973
}

(November 2001)

\begin{abstract}
We point out that, in analogy with spin waves in antiferromagnets, all parameters describing the real-time propagation of soft pions at temperatures below the QCD chiral phase transition can be expressed in terms of static correlators. This allows, in principle, the determination of the soft pion dispersion relation on the lattice. Using scaling and universality arguments, we determine the critical behavior of the parameters of pion propagation. We predict that when the critical temperature is approached from below, the pole mass of the pion drops despite the growth of the pion screening mass. This fact is attributed to the decrease of the pion velocity near the phase transition.
\end{abstract}

Introduction.-Designed for the primary goal of discovering quark-gluon plasma, experiments with collisions of large nuclei, such as those pursued at the Relativistic Heavy-Ion Collider (RHIC) at Brookhaven National Laboratory, also open up possibilities to study strongly interacting matter at extremely high temperatures and densities. In particular, it is hoped that at least some part of the rich phase diagram of QCD can be explored experimentally. On the theoretical side, some problems, such as finding the equation of state of QCD, can be effectively solved by using numerical Monte Carlo techniques. However, many important issues related to real-time behavior and response of high-temperature strongly interacting matter cannot be systematically studied by such methods. This is because lattice techniques rely on the formulation of quantum field theory in imaginary time. As a result, the question of what happens to the hadron spectrum of QCD, which is important, for example, for the understanding of certain features of the dilepton spectrum observed in heavy-ion collisions, cannot be easily answered in a reliable fashion.

Fortunately, many quantities characterizing the real-time behavior of finite-temperature systems can be related, by exact identities, to static (thermodynamic) functions. The most familiar case is the relation between the velocity of sound $u$, the pressure $p$, and the energy density $\epsilon: u=(\partial p / \partial \epsilon)^{1 / 2}$. A less trivial example is that of spin waves in antiferromagnets: it has long been known [1] that at any temperature below the phase transition, at long enough 
wavelengths there exist low-frequency spin waves which have a linear dispersion curve, whose slope is given exactly in terms of static quantities.

In this Letter, we point out that, in thermal QCD, the dispersion relation of soft pions can be determined entirely using static quantities. Such quantities, in principle, can be measured on the lattice. Using this observation, we show that the pion pole mass, which characterizes the propagation of the collective pion modes, decreases as one approaches the critical temperature, despite the well-known fact that the pion screening mass increases in the same limit.

Pion dispersion from static quantities.-From the point of view of symmetry properties, QCD at temperatures $T$ below or just above the temperature of the chiral phase transition $T_{c}$ is similar to a Heisenberg antiferromagnet [2, 3]. With two light quarks $(u$ and $d)$, QCD possesses an approximate chiral $\mathrm{SU}(2)_{V} \times \mathrm{SU}(2)_{A} \simeq \mathrm{O}(4)$ symmetry, which is broken spontaneously to $\mathrm{SU}(2)_{V} \simeq \mathrm{O}(3)$ by the chiral condensate. This is similar to the $\mathrm{O}(3) \rightarrow \mathrm{O}(2)$ symmetry breaking in antiferromagnets. Moreover, the order parameter of QCD, the chiral condensate $\langle\bar{\psi} \psi\rangle$, is distinct from the conserved charges (the vector and axial isospin charges), which makes the real-time behavior of QCD similar to that of antiferromagnets (but not of ferromagnets.)

By analogy with spin waves in antiferromagnets [1], one can show that, at any $T$ below $T_{c}$, the real part of the dispersion relation of soft pions is given by

$$
\omega^{2}=u^{2}\left(\boldsymbol{p}^{2}+m^{2}\right)
$$

provided the quark masses are small enough. We use the following terminology: $u$ is the pion velocity (although it is the velocity only when $m=0$ ), $m$ is the pion screening mass, and the energy of a pion at $\boldsymbol{p}=0, m_{p}=u m$, is the pion pole mass. At zero temperature, $u=1$, and the pole mass coincides with the screening mass. At nonzero temperature, there is no Lorentz invariance, and $u$ generally differs from 1 [4, 5 ]. Such pion modes with modified dispersion relation are termed "quasipions" in Ref. [4].

We shall show that the parameters $u$ and $m$ can be determined by measuring only static (zero-frequency) Euclidean correlators. In particular, $m$ can be extracted from the longdistance behavior of the correlation function of the operator $\pi^{a} \equiv i \bar{\psi} \gamma^{5} \tau^{a} \psi$,

$$
\int d \tau d V e^{-i \boldsymbol{q} \cdot \boldsymbol{x}} \frac{\left\langle\pi^{a}(x) \pi^{b}(0)\right\rangle}{\langle\bar{\psi} \psi\rangle^{2}}=\frac{1}{f^{2}} \frac{\delta^{a b}}{\boldsymbol{q}^{2}+m^{2}},
$$

where $x=(\tau, \boldsymbol{x}), \psi$ is the quark field, $a, b=1,2,3, \tau^{a}$ are isospin Pauli matrices, $\operatorname{Tr} \tau^{a} \tau^{b}=$ $2 \delta^{a b}$, and $\langle\cdots\rangle$ denotes thermal averaging. The integration over the Euclidean time variable $\tau$ is taken in the interval $(0,1 / T)$. In Eq. (2) $\langle\bar{\psi} \psi\rangle$ is the chiral condensate at zero quark masses. Equation (2) also provides the definition of the temperature-dependent pion decay constant $f$.

The pion velocity $u$ is equal to the ratio of the above defined pion decay constant $f$ and the axial isospin susceptibility $\chi_{I 5}$ : 


$$
u^{2}=\frac{f^{2}}{\chi_{I 5}} .
$$

This is a close analog of the equation $c^{2}=\rho_{s} / \chi_{m}$ 피 for the velocity of spin waves in antiferromagnets. The axial isospin susceptibility $\chi_{I 5}$ can be defined as the second derivative of the pressure with respect to the axial isospin chemical potential [see Eq. (5) below], or, equivalently, via the static correlator of the axial isospin charge densities,

$$
\delta^{a b} \chi_{I 5}=\int d \tau d V\left\langle A_{0}^{a}(x) A_{0}^{b}(0)\right\rangle, \quad A_{0}^{a} \equiv \bar{\psi} \gamma^{0} \gamma^{5} \frac{\tau^{a}}{2} \psi
$$

The right hand side of Eq. (田) is free of short-distance divergences in the limit of zero quark masses, when $A_{0}^{a}$ are densities of conserved charges.

The derivation of Eqs. (11)-(田) at non-zero temperature requires an analysis of the hydrodynamic theory similar to the one performed in Ref. [四]. This approach will be presented elsewhere [6]. In this Letter, we use an intuitively simpler (but less rigorous) derivation based on the effective Lagrangian approach. This approach does not allow a correct treatment of dissipative effects, but will be sufficient for our purpose. A somewhat similar approach has been used, for low temperatures, in Ref. [5].

Derivation.- Our strategy is to first write down the most general form of the effective Lagrangian of pions and then relate its free parameters to the correlation functions of QCD by matching the partition function $\mathcal{Z}=e^{\mathcal{P} V / T}$ and its derivatives in the effective and microscopic theories. The quark part of the QCD Lagrangian at finite axial isospin chemical potential $\mu_{I 5}$ is given by:

$$
\mathcal{L}_{\text {quark }}=i \bar{\psi} \gamma^{\mu} D_{\mu} \psi-\left(\bar{\psi}_{L} M \psi_{R}+\text { h.c. }\right)+\mu_{I 5} A_{0}^{3},
$$

where $M=\operatorname{diag}\left(m_{u}, m_{d}\right)$ is the quark mass matrix. The chemical potential $\mu_{I 5}$ is coupled to the axial isospin charge $A_{0}^{3}$ defined in Eq. (4). For simplicity, we set $m_{u}=m_{d}=m_{q}$.

We assume that, in the infrared, the pion thermal width is negligible compared to its energy. This has been seen in explicit calculations at low $T$ [7]. The dynamics of the pions is described, in this case, by some effective Lagrangian $\mathcal{L}_{\text {eff }}$, which we assume to be local, allowing expansion in powers of momenta. This is equivalent to the assumption that the correlation functions have only pole singularities, as in hydrodynamics. To lowest order, the Lagrangian is fixed by symmetries up to three coefficients, $f_{t}, f_{s}$, and $f_{m}$,

$$
\mathcal{L}_{\text {eff }}=\frac{f_{t}^{2}}{4} \operatorname{Tr} \nabla_{0} \Sigma \nabla_{0} \Sigma^{\dagger}-\frac{f_{s}^{2}}{4} \operatorname{Tr} \partial_{i} \Sigma \partial_{i} \Sigma^{\dagger}+\frac{f_{m}^{2}}{2} \operatorname{Re} \operatorname{Tr} M \Sigma,
$$

where $\Sigma$ is an $\mathrm{SU}(2)$ matrix whose phases describe the pions. Because of the lack of Lorentz invariance, $f_{t}^{2}$ and $f_{s}^{2}$ are independent parameters.

The chemical potential $\mu_{I 5}$ enters lowest-order effective Lagrangian (6) via the covariant derivative $\nabla_{0}$ in a way completely fixed by symmetries. This can be seen by promoting the $\mathrm{SU}(2)_{A}$ symmetry in (5) to a local symmetry and treating $\mu_{I 5}$ as the time component of the $\mathrm{SU}(2)_{A}$ vector potential [8]. The covariant derivative $\nabla_{0}$ is forced to have the form 


$$
\nabla_{0} \Sigma \equiv \partial_{0} \Sigma-\frac{i}{2} \mu_{I 5}\left(\tau_{3} \Sigma+\Sigma \tau_{3}\right)
$$

The structure of the Lagrangian (6) is analogous to that of the effective Lagrangian at finite (vector) isospin chemical potential $\mu_{I}$ [9]. A significant difference between the two cases is that the QCD vacuum breaks the $\mathrm{SU}(2)_{A}$ (axial isospin) symmetry spontaneously. It is important to note, however, that the $\mathrm{SU}(2)_{A}$ is a symmetry of the Lagrangian (at $m_{q}=0$ ), as good as the $\mathrm{SU}(2)_{V}$. The conservation of the axial isospin current $A_{\mu}^{a}$ in the chiral limit makes the consideration of finite $\mu_{I 5}$ entirely legitimate.

The pion dispersion relation following from Eq. (6) is given by Eq. (1) with

$$
u^{2}=\frac{f_{s}^{2}}{f_{t}^{2}} \quad \text { and } \quad m^{2}=\frac{m_{q} f_{m}^{2}}{f_{s}^{2}} .
$$

Matching the second derivative of the pressure $\mathcal{P}$ with respect to $\mu_{I 5}$ in QCD and in the effective theory, we find the relation between $f_{t}$ and $\chi_{I 5}$ :

$$
\chi_{I 5}=\frac{\partial^{2} \mathcal{P}}{\partial \mu_{I 5}^{2}}=f_{t}^{2} .
$$

Together with the first of Eqs. (8) and $f=f_{s}$ (see below), this implies Eq. (3). The first derivative with respect to $m_{q}$ gives

$$
-\langle\bar{\psi} \psi\rangle=\frac{\partial \mathcal{P}}{\partial m_{q}}=f_{m}^{2} .
$$

Combining with the second of Eqs. (8), we derive the generalization of the famous GellMann-Oakes-Renner (GOR) relation to finite temperature:

$$
f_{s}^{2} m^{2}=-m_{q}\langle\bar{\psi} \psi\rangle
$$

Finally, we need to show that $f=f_{s}$. We achieve this by treating $M$ as an external field, which we parameterize as $M(x)=m_{q} e^{i \alpha^{a}(x) \tau^{a}}$. Matching derivatives of $\ln \mathcal{Z}$, we find

$$
\begin{aligned}
& \left\langle\pi^{a}(x) \pi^{b}(0)\right\rangle=\frac{\delta^{2} \ln \mathcal{Z}}{m_{q}^{2} \delta \alpha^{a}(x) \delta \alpha^{b}(0)}=f_{m}^{4}\left\langle\phi^{a}(x) \phi^{b}(0)\right\rangle ; \\
& \pi^{a} \equiv i \bar{\psi} \gamma_{5} \tau^{a} \psi, \quad \phi^{a}(x) \equiv \operatorname{ReTr} i \tau^{a} \Sigma(x) / 2 .
\end{aligned}
$$

Note that $\pi^{a}$ is defined in the microscopic theory (QCD), while $\phi^{a}$ is a field of the effective theory.

The correlation function of $\phi^{a}(x)$ can be calculated by expanding the effective Lagrangian in (6) to second order in $\phi^{a}$. We expect the result to match the correlator of $\pi^{a}$ only for small momenta, which means that we have to limit ourselves to zero Matsubara frequency and small spatial momenta, e.g., smaller than the screening mass $m_{\sigma}$ of the order parameter $\sigma=\bar{\psi} \psi$. For the static correlator of $\pi^{a}$, by integrating (12) over $\tau$ and using (10), 


$$
\begin{aligned}
& \int d \tau \frac{\left.\left\langle\pi^{a}(x) \pi^{b}(0)\right)\right\rangle}{\langle\bar{\psi} \psi\rangle^{2}}=\int d \tau\left\langle\phi^{a}(x) \phi^{b}(0)\right\rangle \\
& =\frac{1}{f_{s}^{2}} \int \frac{d^{3} \boldsymbol{q}}{(2 \pi)^{3}} \frac{e^{i \boldsymbol{q} \cdot \boldsymbol{x}} \delta^{a b}}{\boldsymbol{q}^{2}+m^{2}}=\frac{1}{f_{s}^{2}} \frac{e^{-m|\boldsymbol{x}|}}{4 \pi|\boldsymbol{x}|} \delta^{a b} .
\end{aligned}
$$

We see that, by measuring the large-distance $\left(|\boldsymbol{x}| \gg m_{\sigma}^{-1}\right)$ static correlation function of the operator $\pi^{a}=i \bar{\psi} \gamma^{5} \tau^{a} \psi$, we can extract two parameters of the effective Lagrangian (6): the screening mass $m$, and $f_{s}$ which coincides with the decay constant $f$ defined by (2). The third parameter, $f_{t}^{2}$, coincides with the susceptibility $\chi_{I 5}$, which can also be expressed in terms of the static correlation function in Eq. (4). From Eq. (3), we completely determine the dispersion relation of soft pions.

Critical behavior.-For the above results to be valid, pions must be the lightest modes. In particular, this requires $m \ll m_{\sigma}$. If $m_{q}$ is very small, this condition is satisfied everywhere below $T_{c}$, except for a region very close to $T_{c}$. As $T \rightarrow T_{c}$ from below and $m_{\sigma} \rightarrow 0$, one can ask the question: What is the critical behavior of the parameters $u$ and $m$ when $T$ remains sufficiently far from $T_{c}$ so that the hierarchy $m \ll m_{\sigma} \ll T$ is maintained?

Since $u$ and $m$ can be related to static correlation functions, one should expect their critical behavior to be governed by the same static critical exponents known from the theory of critical phenomena. We begin by considering the critical scaling of the decay constant $f=f_{s}$. It is defined via the behavior of a static correlator (13) at distances larger than $m_{\sigma}^{-1}$. In the range of momenta $m \ll|\boldsymbol{q}| \ll m_{\sigma}$ we have [see Eq. (2)]

$$
\int d \tau d V e^{-i \boldsymbol{q} \cdot \boldsymbol{x}}\left\langle\pi^{a}(x) \pi^{b}(0)\right\rangle=\delta^{a b} \frac{\langle\bar{\psi} \psi\rangle^{2}}{f^{2}} \frac{1}{\boldsymbol{q}^{2}} .
$$

On the other hand, at distances short compared to the correlation length, i.e., for momenta such that $m_{\sigma} \ll|\boldsymbol{q}| \ll T$, the correlator of the order parameter $\bar{\psi} \psi$ has the following scaling behavior:

$$
\int d \tau d V e^{-i \boldsymbol{q} \cdot \boldsymbol{x}}\langle\bar{\psi} \psi(x) \bar{\psi} \psi(0)\rangle \sim \frac{1}{|\boldsymbol{q}|^{2-\eta}} .
$$

We also know that in this regime the correlators of $\sigma=\bar{\psi} \psi$ and $\pi^{a}=i \bar{\psi} \gamma^{5} \tau^{a} \psi$ are degenerate, since they are related by the $\mathrm{SU}(2)_{A}$ symmetry, which is restored at $T_{c}$. Thus the correlator (15) must match with the correlator (14) at the scale $|\boldsymbol{q}| \sim m_{\sigma}$. This requires

$$
f^{2}=A m_{\sigma}^{-\eta}\langle\bar{\psi} \psi\rangle^{2}
$$

The coefficient $A$ cannot be found from scaling arguments but is finite and regular at $T_{c}$. The exponent $\eta$ in the universality class of the $\mathrm{O}(4)$ sigma-model in $d=3$ dimensions (to which two-flavor QCD at $T_{c}$ belongs [2]) is known: $\eta \approx 0.03$ (see, e.g., [10,3]).

At $T \rightarrow T_{c}$ the scaling laws for the inverse correlation length $m_{\sigma}$ and the order parameter $\langle\bar{\psi} \psi\rangle$ are also known from universality, 


$$
\begin{aligned}
m_{\sigma} & \sim t^{\nu}, \\
\langle\bar{\psi} \psi\rangle & \sim t^{\beta},
\end{aligned}
$$

where $t=\left(T_{c}-T\right) / T_{c}$. Thus we find

$$
f^{2} \sim t^{2 \beta-\nu \eta}=t^{(d-2) \nu}
$$

where in the last equation the relation

$$
2 \beta=\nu(d-2+\eta)
$$

is used (recall that all scaling exponents can be expressed in terms of two independent ones, e.g., $\eta$ and $\nu$ ). From this point on, we set $d=3$, so $f \sim t^{\nu / 2}$. The is the same as the Josephson scaling for the superfluid density in helium [11]. Contrary to a naive expectation, $f$ scales differently from the order parameter $\langle\bar{\psi} \psi\rangle \sim t^{\beta}$. The difference is numerically small, due to the smallness of $\eta$. In the $\mathrm{O}(4)$ universality class in $d=3, \nu \approx 0.73$ and $\beta \approx 0.38$ [10].

Next, we point out that $\chi_{I 5}$ is finite at $T=T_{c}$, where it is degenerate with the vector isospin susceptibility. The singular behavior of $\chi_{I 5}$ is dominated by the mixing of $A_{0}^{a}$ with operators linear or quadratic in $\sigma$ or $\pi^{a}$ in the dimensionally reduced theory describing infrared modes $|\boldsymbol{q}| \ll T$. Such a mixing, however, is forbidden by the $\mathrm{O}(4)$ chiral symmetry, as well as by charge conjugation. This is consistent with the lattice result that the vector isospin susceptibility is finite at $T_{c}$ [12]. Since $f_{t}^{2}=\chi_{I 5}$, finiteness of $\chi_{I 5}$ invalidates a common assumption that $f_{t} \rightarrow 0$ at $T_{c}$. Note that above $T_{c}$ there are no propagating soft pion modes, so the parameters of of the effective Lagrangian (such as $f_{t}$ ), and hence Eq. (9)), lose their meaning, even as $\chi_{I 5}$ remains well defined and finite. This is not surprising if one recalls that, as $T \rightarrow T_{c}$ from below, the domain of validity of the Lagrangian $(6)\left(|\boldsymbol{q}| \ll m_{\sigma}\right)$ shrinks away and disappears at $T_{c}$.

Now we are ready to find the scaling of $u$. Using Eq. (3), the scaling of $f$ in Eq. (19), and the fact that $\chi_{I 5}$ is finite at $T_{c}$, we find

$$
u^{2} \sim f^{2} \sim t^{\nu}
$$

This means that the velocity of pions vanishes at $T_{c}$.

The scaling of the screening mass $m$ can be found from the GOR relation (11) (recall that $m_{q}$ is assumed to be small):

$$
m^{2}=-\frac{m_{q}\langle\bar{\psi} \psi\rangle}{f^{2}} \sim m_{q} t^{\beta-\nu}
$$

In the $O(4)$ universality class $\beta<\nu$, which implies that the static screening pion mass grows (at fixed $m_{q} \neq 0$ ) as $T \rightarrow T_{c}$. This fact is already known from lattice simulations of QCD.

The pole mass of the pion, $m_{p}$, scales differently: 


$$
m_{p}^{2} \equiv u^{2} m^{2}=-\frac{m_{q}\langle\bar{\psi} \psi\rangle}{\chi_{I 5}} \sim m_{q} t^{\beta} .
$$

This means the pole mass of the pion drops as $T \rightarrow T_{c}$.

For the formulas (21)-(23) to be valid it is necessary that $t \ll 1$. However, for any $m_{q} \neq 0$, these formulas break down when $t$ is so small that the condition $m \ll m_{\sigma}$ is violated. Using Eqs. (17) and (22), we see that this happens when $t \sim m_{q}^{1 / \beta \delta}$ or smaller. In the regime $t \ll m_{q}^{1 / \beta \delta}$ the "distance" from the critical point $\left(T=T_{c}, m_{q}=0\right)$ is controlled by $m_{q}$, but not $t$. The $m_{q}$ scaling of all quantities can be obtained starting from

$$
\langle\bar{\psi} \psi\rangle \sim m_{q}^{1 / \delta} \quad \text { at } t=0 .
$$

Comparing this to Eq. (18), we see that $t$ and $m_{q}^{1 / \beta \delta}$ have the same scaling dimension. Using the scaling hypothesis we can easily obtain the $m_{q}$ scaling by replacing $t$ with $m_{q}^{1 / \beta \delta}$. For example,

$$
m^{2} \sim m_{q}^{1-(\nu-\beta) / \beta \delta}, \quad m_{p}^{2} \sim m_{q}^{1+1 / \delta} .
$$

$\left(m_{p}\right.$ now has the meaning of the typical frequency of the pion mode with zero momentum. This mode may be overdamped in this regime.) Both masses vanish as $m_{q} \rightarrow 0$ at $T=T_{c}$; however, for the screening mass $m^{2} \gg m_{q}$, while for the pole mass $m_{p}^{2} \ll m_{q}$. In particular, near the phase transition $m_{p} \ll m$.

The decrease of the pion pole mass may have interesting consequences for heavy-ion collisions. It is the pole mass of a hadron, rather than its static screening mass, that affects the observed spectrum. Within statistical models for hadron production, the drop in the pion pole mass would lead to an overpopulation of pions at low momenta, provided the chemical freezeout temperature $T_{\mathrm{ch}}$, at which the hadron abundances are fixed, is close to $T_{c}$. For a crude estimate of this effect we use $\Lambda_{\mathrm{QCD}} \sim 200 \mathrm{MeV}$ as the typical QCD scale in Eq. (25), and $T_{\mathrm{ch}} \sim 170 \mathrm{MeV}$ as an estimate for the freezeout temperature [13, which is indeed very close to $T_{c}$. The shift of the pole mass near $T_{c}$ is approximately $\Delta m \equiv m_{p}-m_{\pi} \approx m_{\pi}\left(\left(m_{q} / \Lambda_{\mathrm{QCD}}\right)^{1 / 2 \delta}-1\right) \approx-0.3 m_{\pi}$, where $\delta \approx 5$, and the pion multiplicity at small momenta is enhanced by roughly $\exp \left(-\Delta m / T_{\mathrm{ch}}\right) \approx 1.3$. This is a noticeable effect, although it is smaller than the known contribution to pion overpopulation due to the feeddown from the decays of resonances [13]. This enhancement is comparable to the effect of the pion chemical potential $\mu_{\pi} \sim 50 \mathrm{MeV}$ induced by pion kinetics after the chemical freezeout [14].

Another potential consequence of the fact that pion velocity decreases at $T_{c}$ is the possibility of Cherenkov radiation of pions by a hard probe moving through the hot medium created in a heavy-ion collision.

The authors are indebted to D. Bödeker, N. Christ, R. Mawhinney, L. McLerran, A. Mueller, and R. Pisarski for discussions. We thank RIKEN, Brookhaven National Laboratory, and U.S. Department of Energy [DE-AC02-98CH10886] for providing the facilities essential for the completion of this work. The authors are supported, in part, by DOE OJI grants. The work of D.T.S. is supported, in part, by the Alfred P. Sloan Foundation. 


\section{REFERENCES}

[1] B. I. Halperin and P. C. Hohenberg, Phys. Rev. 188, 898 (1969); P. C. Hohenberg and B. I. Halperin, Rev. Mod. Phys. 49, 435 (1977).

[2] R. D. Pisarski and F. Wilczek, Phys. Rev. D 29, 338 (1984).

[3] K. Rajagopal and F. Wilczek, Nucl. Phys. B399, 395 (1993) hep-ph/9210253.

[4] E. V. Shuryak, Phys. Rev. D 42, 1764 (1990).

[5] R. D. Pisarski and M. Tytgat, Phys. Rev. D 54, R2989 (1996) hep-ph/9604404.

[6] D. T. Son and M. A. Stephanov, hep-ph/0204226.

[7] J. L. Goity and H. Leutwyler, Phys. Lett. B 228, 517 (1989).

[8] J. B. Kogut, M. A. Stephanov, and D. Toublan, Phys. Lett. B 464, 183 (1999) hepph/9906346]; J. B. Kogut, M. A. Stephanov, D. Toublan, J. J. Verbaarschot, and A. Zhitnitsky, Nucl. Phys. B582, 477 (2000) hep-ph/0001171.

[9] D. T. Son and M. A. Stephanov, Phys. Rev. Lett. 86, 592 (2001) hep-ph/0005225]; Phys. At. Nucl. 64, 834 (2001) [Yad. Fiz. 64, 899 (2001)] [hep-ph/0011365.

[10] G. A. Baker, B. G. Nickel and D. I. Meiron, Phys. Rev. B 17, 1365 (1978).

[11] B. D. Josephson, Phys. Lett. 21, 608 (1966).

[12] S. Gottlieb, W. Liu, D. Toussaint, R. L. Renken, and R. L. Sugar, Phys. Rev. Lett. 59, 2247 (1987); S. Gottlieb, U. M. Heller, A. D. Kennedy, S. Kim, J. B. Kogut, C. Liu, R. L. Renken, D. K. Sinclair, R. L. Sugar, D. Toussaint, and K. C. Wang, Phys. Rev. D 55, 6852 (1997) hep-lat/9612020.

[13] P. Braun-Munzinger, J. Stachel, J. P. Wessels, and N. Xu, Phys. Lett. B 344, 43 (1995) nucl-th/9410026; Phys. Lett. B 365, 1 (1996) nucl-th/9508020; P. Braun-Munzinger, I. Heppe, and J. Stachel, Phys. Lett. B 465, 15 (1999) nucl-th/9903010.

[14] C. M. Hung and E. V. Shuryak, Phys. Rev. C 57, 1891 (1998) hep-ph/9709264. 